\title{
Protective Effects of Glucagon-Like Peptide-1 Analog on Renal Tubular Injury in Mice on High-Fat Diet
}

\author{
Honglei Guo ${ }^{a}$ Hongmei Lia Bin Wang ${ }^{b}$ Wei Ding ${ }^{c} \quad$ Lilu Ling ${ }^{a}$ Min Yang ${ }^{a}$ \\ Jianying Niu ${ }^{a}$ Yong Gu
}

${ }^{a}$ Division of Nephrology, The Fifth People's Hospital of Shanghai, Fudan University, ${ }^{\text {bDivision of }}$ Nephrology, Huashan Hospital and Institute of Nephrology, Fudan University, 'Division of Nephrology, Shanghai Ninth People's Hospital, School of Medicine, Shanghai Jiaotong University, Shanghai, China

\section{Key Words}

GLP-1 • ERS • Apoptosis • AT1R • Renal tubular

\begin{abstract}
Aims: The study aimed to investigate the renoprotective effect of glucagon-like peptide-1 (GLP-1) against renal tubular injury in C57BL/6 mice induced by a high-fat diet (HFD). Methods: Twenty C57BL/6 mice were fed HFD for 12 weeks. Ten of these mice were treated with GLP-1 at $200 \mu \mathrm{g} / \mathrm{kg}$ subcutaneously twice daily for 4 weeks (HFDG group), and the other ten mice received vehicle only (HFD group). Ten mice fed standard rodent chow served as controls (Con group). Body weight, kidney weight, food intake, and systolic blood pressure were measured. The expression of endoplasmic reticulum stress (ERS) markers (BIP, p-eIF2 $\alpha$, ATF4, and CHOP) and apoptosis in the kidney were examined utilizing western blotting, immunohistochemistry and TUNEL, respectively. Angiotensin II and angiotensin II type 1 receptor (AT1R) were examined by ELISA. Human proximal tubule epithelial cells (HK2) were treated with GLP-1(150 $\mathrm{nM}$ ) followed by treatment with palmitic acid (500 nM [PA]) for $24 \mathrm{~h}$. HK2 cells treated with BSA were used as controls. The protein levels of ERS markers, apoptosis-associated protein, and AT1R were measured by western blotting. Results: Increase of body weight, food intake, and systolic blood pressure was less pronounced in GLP-1 treated HFDG mice compared to HFD mice. The levels of ERS markers (BIP, p-eIF2 $\alpha$, ATF4, and CHOP) and apoptosis decreased following GLP-1 treatment in vivo and in vitro $(p<0.05)$. Increased AT1R induced by HFD and PA were blocked with GLP-1 treatment. In contrast, the level of angiotensin II after GLP-1 treatment was not significantly different between the HFD and HFDG mice. Conclusion: The study indicated that saturated fatty acids induced ERS and apoptosis in the kidney and increased AT1R expression. GLP-1 treatment exerted renoprotective effects against saturated fatty acid-induced kidney tubular cell ERS and apoptosis together with inhibition of AT1R expression in vivo and in vitro.
\end{abstract}




\section{Introduction}

Accumulation of saturated fatty acids in the kidney is associated with obesity-related glomerulopathy, including glomerular hyperfiltration, tubulointerstitial inflammation, proximal tubular cell apoptosis, and renal fibrosis [1-3]. Obesity-related inflammation and adipokine deregulation is responsible for kidney function, as demonstrated in rodent models [4]. Clinical studies have also indicated a direct relationship between obesity and renal complications [5], and an association between the degree of obesity and chronic kidney disease [6]. Increased intrarenal levels of fatty acids can induce oxidative and endoplasmic reticulum stress (ERS), thus activation of the unfolded protein response and apoptosis [7].

The 78-kDa glucose regulated protein (BIP), an ER resident chaperone, enhances newly synthesized folded proteins, decreases the accumulation of misfolded proteins, and promotes the ER-associated protein degradation which is increased in obesity [3]. Deteriorated ERS activates protein kinase-like endoplasmic reticulum kinase and induces phosphorylation of eukaryotic initiation factor (eIF2 $\alpha$ ), resulting in up-regulated activating transcription factor 4 (ATF4) and the transcription factor, C/EBP homologous protein (CHOP), thus initiating cell apoptosis [8]. Excessive ERS induced by saturated fatty acid activates CHOP-mediated up-regulated apoptotic Bax, and down-regulates anti-apoptotic Bcl-2. Previous evidence has shown that activated ERS in the kidney of high-fat diet (HFD) mice induces renal injury related to podocytes, proximal tubule cell apoptosis, and mesangial cells [1, 3, 9].

Plasma angiotensin II levels are elevated in obesity [10], resulting in oxidative stress and apoptosis [11]. Angiotensin II is recognized to induce a range of injuries, such as fibrogenesis, oxidative stress, ERS, and apoptosis throughout angiotensin II receptor (AT1R). Cellular oxidative stress induced by activation of the AT1R can be reversed by oral AT1 receptor blocker in the $\beta$-cell line, MIN6 [12], and mice lacking AT1R exhibit attenuation of diet-induced obesity and insulin resistance [13], indicating that AT1R plays a key role in obesity-induced renal injury.

Glucagon-like peptide-1(GLP-1), a gut incretin hormone, is used for type 2 diabetes and obesity. GLP-1 can decrease hyperglycemia, improve beta cell growth, reduce food intake and body weight [14], and has anti-ERS effects [15]. A clinical study reported that patients with type 2 diabetes have significantly lower levels of the predominant circulating saturated free fatty acid following a GLP-1 subcutaneous infusion, with $2 \mathrm{~kg}$ of weight loss [16]. GLP-1 receptor (GLP-1R) has been confirmed to exist in the proximal tubules based on mRNA and protein levels. The renoprotective effect of diabetic nephropathy and acute kidney injury by GLP-1 therapy is associated with inhibition of renal inflammation and oxidative stress rather than metabolic improvement [17]. Inhibition of dipeptidyl peptidase-4, thus cleaving GLP-1, is a renoprotective effect of cisplatin-induced renal injury and rodent ischemia-reperfusion injury models through anti-inflammation and anti-apoptosis by lowering the mRNA expression ratios of Bax:Bcl-2 and Bim:Bcl-2 [18, 19]. GLP-1 has been shown to prevent apoptosis in diabetic kidney disease and ischemia-reperfusion injury models [18]. Whether or not this renoprotection is involved in the obesity-related renal disease inhibition of ERS and apoptosis by blocking AT1R is unclear. The present study was conducted to explore the effectiveness of GLP-1 on renal tubular ERS and apoptosis induced in mice fed HFD and palmitic acid (PA)-incubated HK2 cells, and to investigate the underlying mechanism.

\section{Materials and Methods}

\section{Materials}

PA, BSA, and GLP-1 were purchased from Sigma-Aldrich (St. Louis, MO, USA). Anti-CHOP, anti-BIP, anti-p-eIF2 $\alpha /$ eIF $2 \alpha$, anti-ATF4, and anti-cleaved caspase 3 were purchased from Cell Signaling Technology (Beverly, MA, USA). Anti-AT1R, anti-sodium-glucose cotransporter 2 (SGLT2), Anti-Bcl-2, anti-Bax, antiGAPDH, and anti- $\beta$-actin were from Abcam (Cambridge, MA,USA). Terminal deoxynucleotidyl transferasemediated dUTP-biotin nick end labeling (TUNEL) was obtained from Roche(Switzerland). The angiotensin II radioimmunoassay kit was bought from Mercodia (Uppsala, Sweden). 


\section{Cellular Physiology Cell Physiol Biochem 2017;41:1113-1124 \begin{tabular}{ll|l|l|l|l} 
DOI: 10.1159/000464118 & 2017 The Author(s). Published by S. Karger AG, Basel \\
and Biochemistry & Published online: February 28, 2017 www.karger.com/cpb
\end{tabular}}

Guo et al.: GLP-1 Ameliorates Renal Injury

Animals

All animal procedures were reviewed and approved by the Institutional Animal Care and Use Committee at Fudan University (Shanghai, China). Six-week-old male C57BL/ 6 mice were obtained from the Experimental Animal Center of Fudan University and were housed on a $12 \mathrm{~h}$ light-dark cycle. The mice were fed with free access to standard rodent chow (10\% of total calories from fat) and water or HFD (60\% of total calories from fat) for 12 weeks. The mice were divided into three groups ( $n=10$ per group), as follows: control with standard diet (Con); high-fat diet group (HFD); and high-fat diet with GLP-1 treatment (HFDG). GLP-1 was administered subcutaneously $(200 \mu \mathrm{g} / \mathrm{kg}$, twice daily for 4 weeks). HFD received normal saline alone. Body weight and food intake were measured at 12 and 16 weeks. The food intake of ten mice in each group caged separately was measured for 5 days before intervention and at the end of the GLP-1 infusion. At week 16, all mice were anesthetized with pentobarbital, and the kidneys were rapidly excised.

\section{Cell culture}

The immortalized human proximal tubular cell line (HK2) was cultured in 60-mm dishes. HK2 was cultured in DMEM/F12, supplemented with $10 \%$ fetal bovine serum in an atmosphere of $5 \% \mathrm{CO}_{2}$ in air at $37^{\circ} \mathrm{C}$. HK2 were starved overnight in culture medium without fetal bovine serum prior to treatment with PA (500 nM) or GLP-1(150 nM) for $24 \mathrm{~h}$. HK2 cells with BSA served as controls. Then, HK2 were fixed by $4 \%$ paraformaldehyde for immunofluorescence or lysed with RIPA buffer and a protease inhibitor cocktail for western blotting. HK2 cells grown on glass cover slips in the different groups were stained with Hochest33258 and viewed by fluorescence microscopy for the measurement of apoptosis. An overexpression plasmid AT1R-pEX-3 containing the full-length AT1R gene transiently transfected into the HK2 cells was constructed by JK Genome Corporation, China.

\section{Western blotting}

The kidney tissues were homogenized and the supernatant was collected after centrifugation at $12,000 \mathrm{~g}$ for $20 \mathrm{~min}$ at $4^{\circ} \mathrm{C}$. We separated the lysates on $10 \%$ polyacrylamide gels before immunoblotting. The protein concentrations of the tissues were detected by the BCA method (Beyotime, Shanghai, China). Protein samples were added in the SDS-PAGE gel and transferred to polyvinylidene difluoride membranes. Membranes were blocked for $1 \mathrm{~h}$ at room temperature with 3\% bovine serum albumin. The membranes were incubated with the primary antibodies at a dilution of 1:1000. An ECL advance system (Amersham, Little Chalfont, UK) was used to analyze the membranes.

\section{Immunohistochemistry and TUNEL}

Kidney samples were fixed with $4 \%$ paraformalin for $24 \mathrm{~h}$. After graded alcohol dehydration, kidney samples were embedded with paraffin, then cut in 4- $\mu$ m sections for immunostaining of SGLT2, caspase 3, AT1R, and TUNEL. Tissue sections were dewaxed and rehydrated, then the antigens were unmasked by microwave pre-treatment in citrate buffer. Sections were blocked with $5 \%$ fetal bovine serum for $1 \mathrm{~h}$ at room temperature, then incubated overnight at $4^{\circ} \mathrm{C}$ with antibodies $\left.11: 200\right)$. Slides were rinsed in phosphate-buffered saline 3 times for 5 min and exposed to the secondary antibody for $1 \mathrm{~h}$. Finally, slides were incubated with $\mathrm{ABC}$ complex for $30 \mathrm{~min}$ at $37^{\circ} \mathrm{C}$ and bound peroxidase activity was measured with DAB. Slides were imaged with a Nikon Eclipse 80i Epi-fluorescence microscope equipped with a digital camera (DS-Ri1; Nikon, Japan).

TUNEL assay was chosen to detect apoptotic cell death with a TUNEL system kit. Green-labeled TUNEL-positive cells were counted under $400 \times$ magnification in at least 10 random fields. The percentage of TUNEL-positive cells/total number of HK2 cells represented the apoptotic index. Blinding was implemented in all counting procedures.

\section{Immunofluorescence}

HK2 cells were seeded onto sterile glass cover slips in 24-well dishes for $36 \mathrm{~h}$. HK2 were treated with PA (500 nmol) and GLP-1(150 nM) for $24 \mathrm{~h}$. Cells were fixed with $0.3 \mathrm{ml}$ of 4\% paraformaldehyde for $15 \mathrm{~min}$ prior to permeabilization in $0.25 \%$ Triton X-100 for $10 \mathrm{~min}$ at room temperature. Cells were blocked with $5 \%$ fetal bovine serum for $1 \mathrm{~h}$ at room temperature followed by incubation with primary and secondary antibodies. Primary antibodies were detected with fluorescence-labeled anti-mouse and anti-rabbit antibodies diluted 1:200 (Beyotime, Haimen, China). Nuclei were counterstained with 4',6-diamidino-2- 
phenylindole (DAPI,H-1200, Vector Laboratories, Burlingame, CA, USA) and were coverslipped on slides and imaged by a confocal fluorescent microscope (Nikon DS-Ri, Japan). Quantification was performed in a blinded manner. The AT1R immunofluorescence intensity was quantified by Image J software by measuring the positive staining area.

Statistical analyses

The results are expressed as the mean \pm SD. Data were analyzed by one-way ANOVA. A statistically significant difference was set at $p<0.05$.

\section{Results}

GLP-1 decreased food intake and ameliorated metabolic data

Food intake declined significantly in HFDG mice compared with HFD mice $(42.8 \pm 9.7 \mathrm{vs}$. $56.9 \pm 8.1, p<0.05)$. The body weight and kidney weight in HFD mice were remarkably higher than Con mice at the end of the intervention. After GLP-1 treatment, the reduction in body and kidney weights were significantly compared with HFD mice $(33.7 \pm 7.8 \mathrm{~g}$ vs. $47.9 \pm 9.6 \mathrm{~g}$ and $0.28 \pm 0.015 \mathrm{~g} v s .0 .30 \pm 0.009 \mathrm{~g}$, respectively, $p<0.05$ ). Systolic blood pressure(SBP) was higher in HFD mice than Con mice $(121 \pm 17.6 \mathrm{mmHg} v s .98 \pm 10.8 \mathrm{mmHg}, p<0.05)$, and GLP-1 treatment significantly decreased SBP $(106 \pm 19.8 \mathrm{mmHg})$, as shown in Fig. 1.

\section{GLP-1 attenuated renal tubular injury in HFD mice}

The histologic findings of renal tubules were assessed through periodic acid-Schiff staining (PAS). Representative photomicrograghs illustrated vacuolated tubular cells completely filled in the cortex of the HFD mice. The vacuoles pushed the nucleus to the edge of the proximal tubular cells. Chronic GLP-1 treatment remarkably suppressed the development of vacuolated tubular cells, while the injury attenuated responses to GLP-1 treatment (Fig. 2A). SGLT2 is mainly located in the brush border membrane of the proximal convoluted tubule of the kidney. SGLT2 expression was less extensive in HFD mice compared with Con mice, while SGLT2 expression in the proximal tubules was restored in HFDG mice as demonstrated by immunohistochemistry (Fig. 2B and C) and western blotting (Fig. 2D and E).

As shown in Fig. 3, western blotting demonstrated remarkably increased expression of BIP, p-eIF2 $\alpha$, ATF 4, and CHOP proteins in the cortex of HFD mice compared to the Con group and GLP-1 decreased the increasing expression $(p<0.05)$.

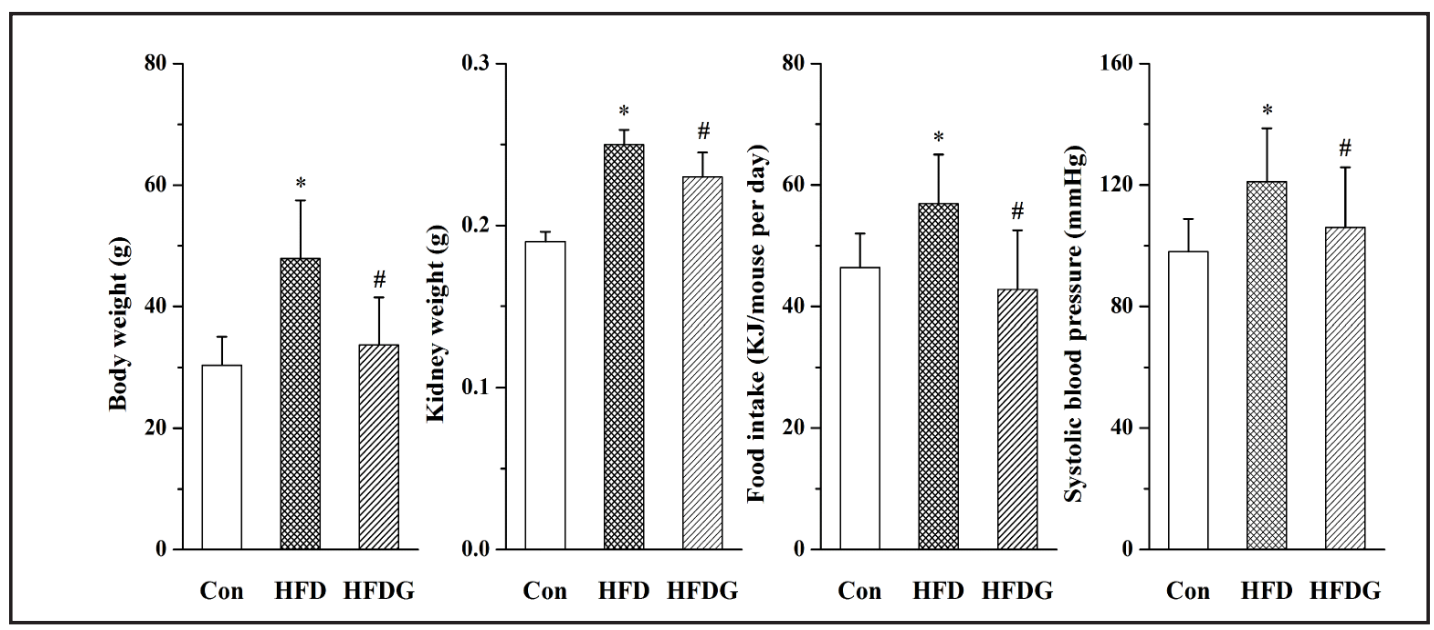

Fig. 1. Effect of GLP-1 treatment on body weight, kidney weight, food intake and systolic blood pressure. A. Body weight of the three groups, $n=10$ per group. B. Bilateral kidney weight was measured. C. Food intake of ten mice in each group caged separately was measured. D. Systolic blood pressure was measured three times by Tail-cuff and the data was averaged. " $p<0.05$ compared with Con; ${ }^{*} p<0.05$ compared with HFD.

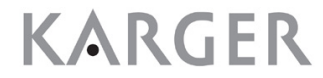




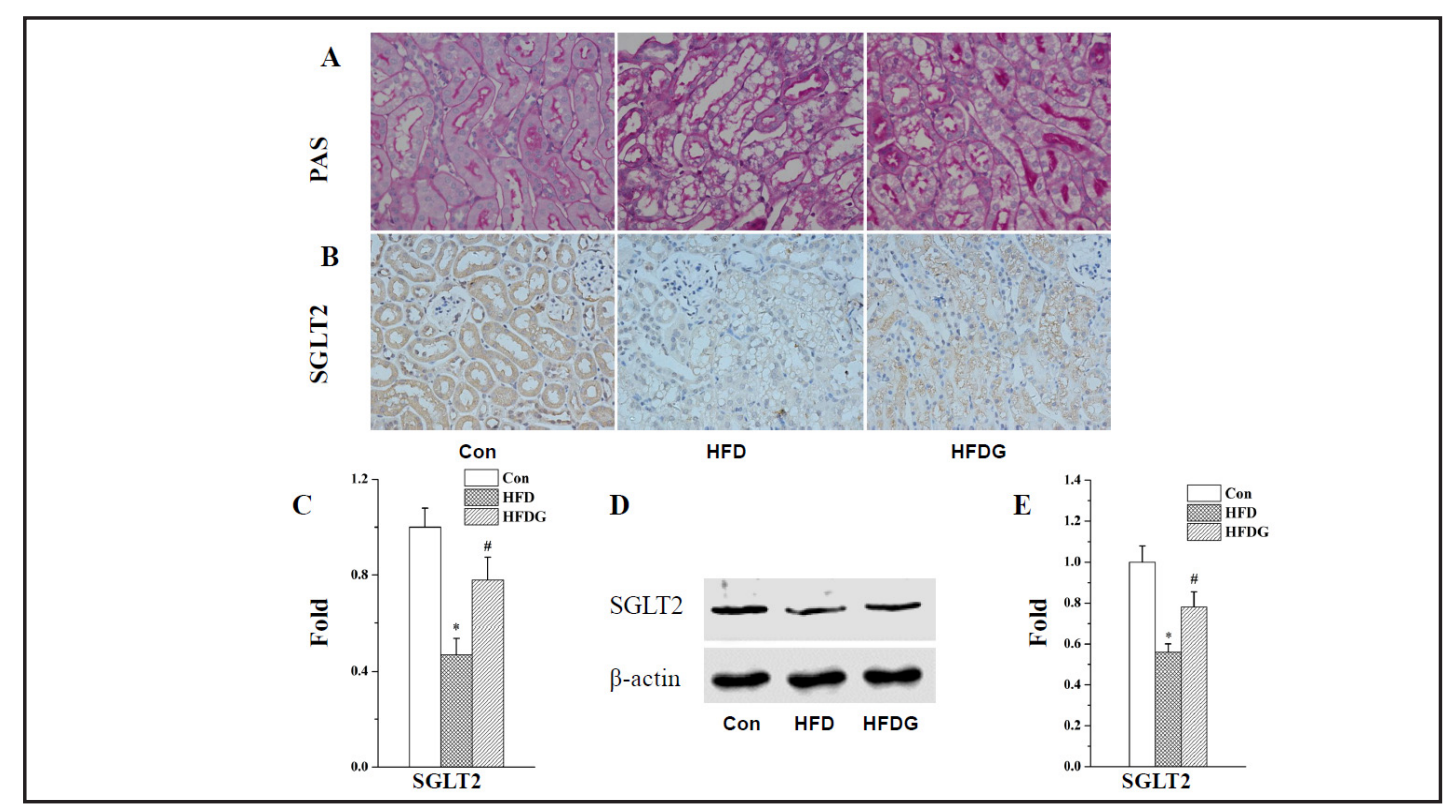

Fig. 2. GLP-1 treatment protects renal injury induced by the high-fat diet in mice. A. Periodic Acid-Schiff staining(PAS) illustrated that vacuolated proximal convoluted tubular cells and impaired brush border induced by HFD were ameliorated with GLP-1 treatment. B. Immunostaining of sodium-glucose cotransporter 2 (SGLT2) existed in cytoplasm of proximal tubular cells in Con mice. In HFD mice immunolabeling of SGLT2 was seen less extensive than Con and HFDG mice in proximal tubule cells. Quantitative assessment of SGLT2 was shown in C. D and E. Western blots analysis of SGLT2 expression. High-fat diet treatment was associated with decreased levels of SGLT2 protein expression in the kidney cortex. This was attenuated by GLP-1. ${ }^{*} p<0.05$ compared with Con; ${ }^{*} p<0.05$ compared with HFD.

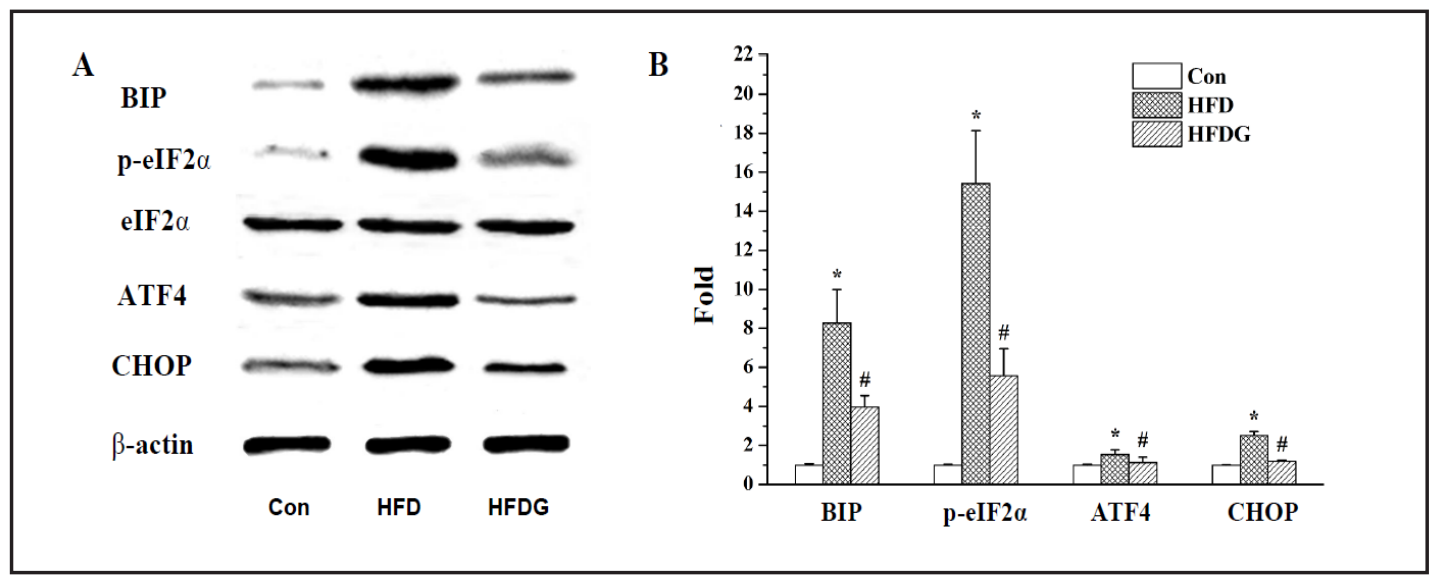

Fig. 3. GLP-1 treatment inhibits ERS in the high-fat diet mice. A. Western blots analysis of increased ERS markers (BIP, p-eIF2 $\alpha$, ATF4 and CHOP proteins) of the HFD mice was ameliorated with GLP-1 treatment in the cortex of HFD mice. B. Corresponding densitometric analyses of levels of ERS markers normalized to $\beta$-actin. ${ }^{*} p<0.05$ compared with Con; ${ }^{\#} p<0.05$ compared with HFD.

The anti-apoptosis protein, Bcl-2, was inhibited in HFD mice compared with Con mice, while Bcl-2 was markedly increased in HFDG mice. Bax and cleaved caspase 3 were higher in HFD mice than Con mice; however, GLP- 1 treatment inhibited all of the changes $(p<0.05$; Fig. $4 \mathrm{~A}$ and $\mathrm{B}$ ). In the kidneys of Con mice, few positive cells of apoptosis were detected in the tubules. In HFD mice, HFD was associated with a significantly increased number of apoptotic cells in renal tubules compared with Con mice (Fig. 4C, 4D, 4E and F). In contrast, apoptotic cells were markedly decreased when HFD mice were treated with GLP-1 $(p<0.05)$. 


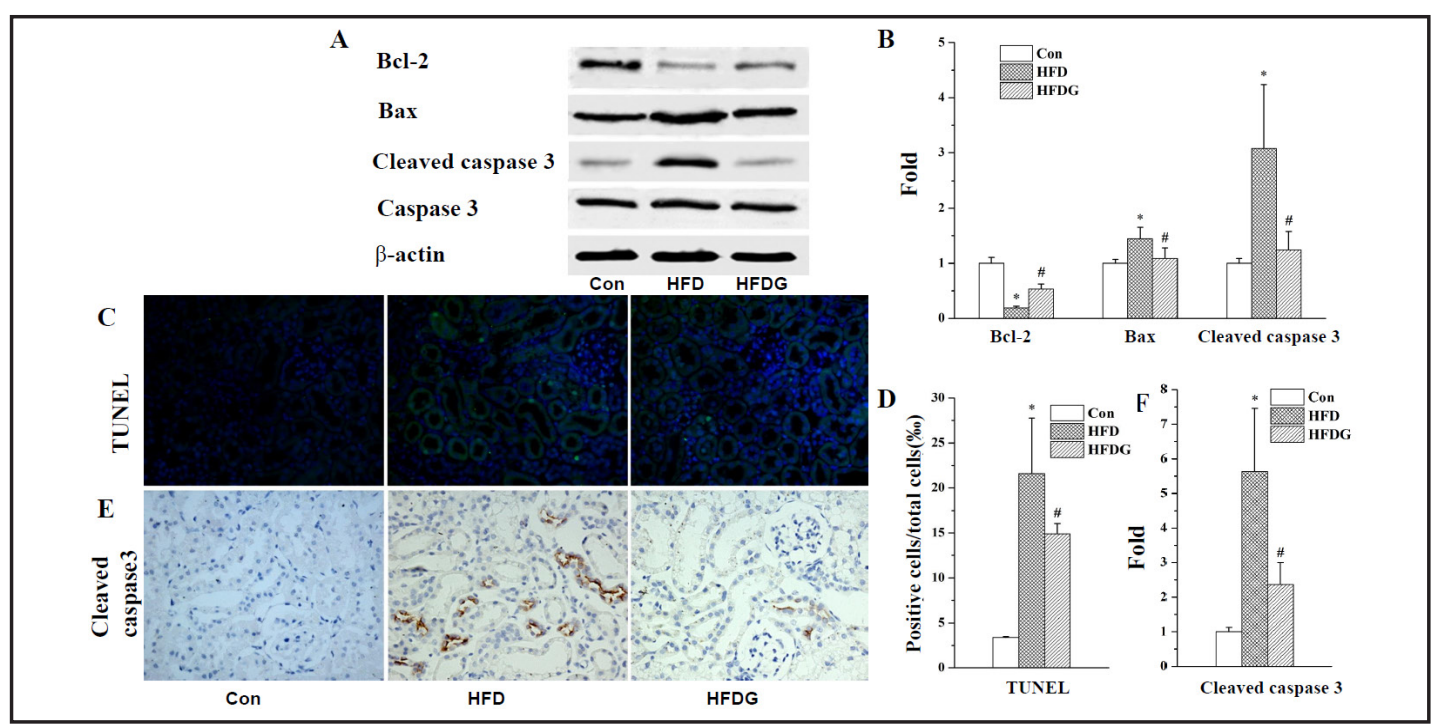

Fig. 4. GLP-1 treatment prevents apoptosis in the high-fat diet mice. A. Western blots analysis of high fat diet-induced decreased Bcl-2, and increased Bax and cleaved caspase-3 protein steady-state levels in kidney tubular cells, which were attenuated by GLP-1 treatment. B. Corresponding densitometric analyses of levels of Bcl-2, Bax and cleaved caspase-3 protein normalized to $\beta$-actin. C. Representative TUNEL staining of renal tubular cells. Apoptotic nuclei were observed. D. Immunostaining of caspase 3 existed in cytoplasm of proximal tubular cells. E. The percentage of apoptotic cells (TUNEL-positive cells) was obtained in at least 10 fields from the three groups. Corresponding quantitative analysis indicated that GLP-1 treatment decreased the apoptotic tubular cells. F. Corresponding quantitative analysis demonstrated that GLP-1 treatment decreased the caspase 3 expression in kidney tubular cells compared to HFD mice. ${ }^{*} p<0.05$ compared with Con; ${ }^{\#} p<0.05$ compared with HFD.

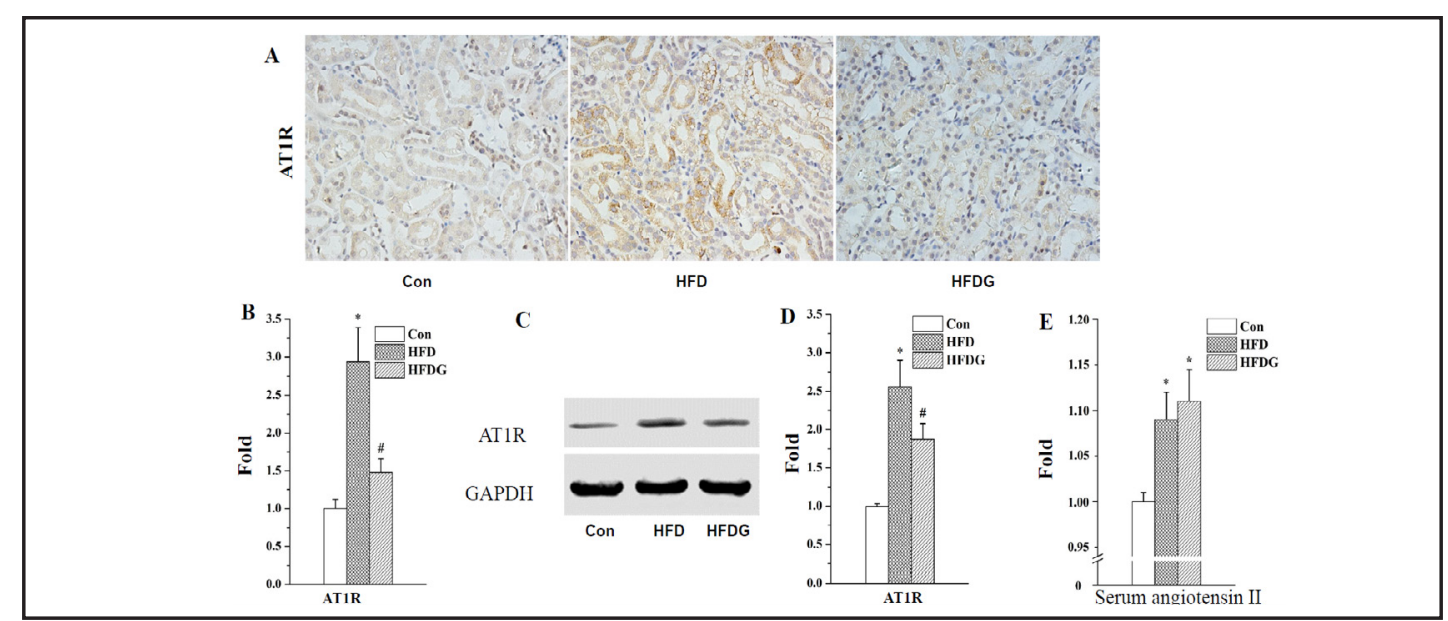

Fig. 5. GLP-1 treatment blocks AT1R expression in the renal tubular cells. A and B. Immunostaining of AT1R expression located in cytoplasm of proximal tubular cells. Corresponding quantitative analysis demonstrated that GLP-1 treatment decreased the AT1R expression in kidney tubular cells compared to HFD mice. C. Western blot analysis of high fat diet-induced increased AT1R expression in the kidney tubular cells, which were deceased by GLP-1 treatment. D. Corresponding densitometric analyses of levels of AT1R protein normalized to GAPDH. E. Serum levels of angiotensin II measured by ELISA. * $p<0.05$ compared with Con; \# $p<0.05$ compared with HFD.

\section{GLP-1 inhibited AT1R expression in renal tubules of mice fed HFD}

Mice fed HFD were associated with an increase in AT1R expression in the renal tubules. In the kidneys of GLP-1-treated mice, positive staining for AT1R was significantly decreased $(p<0.05)$, as shown in Fig. 5A and B. Western blotting showed that AT1R expression was 


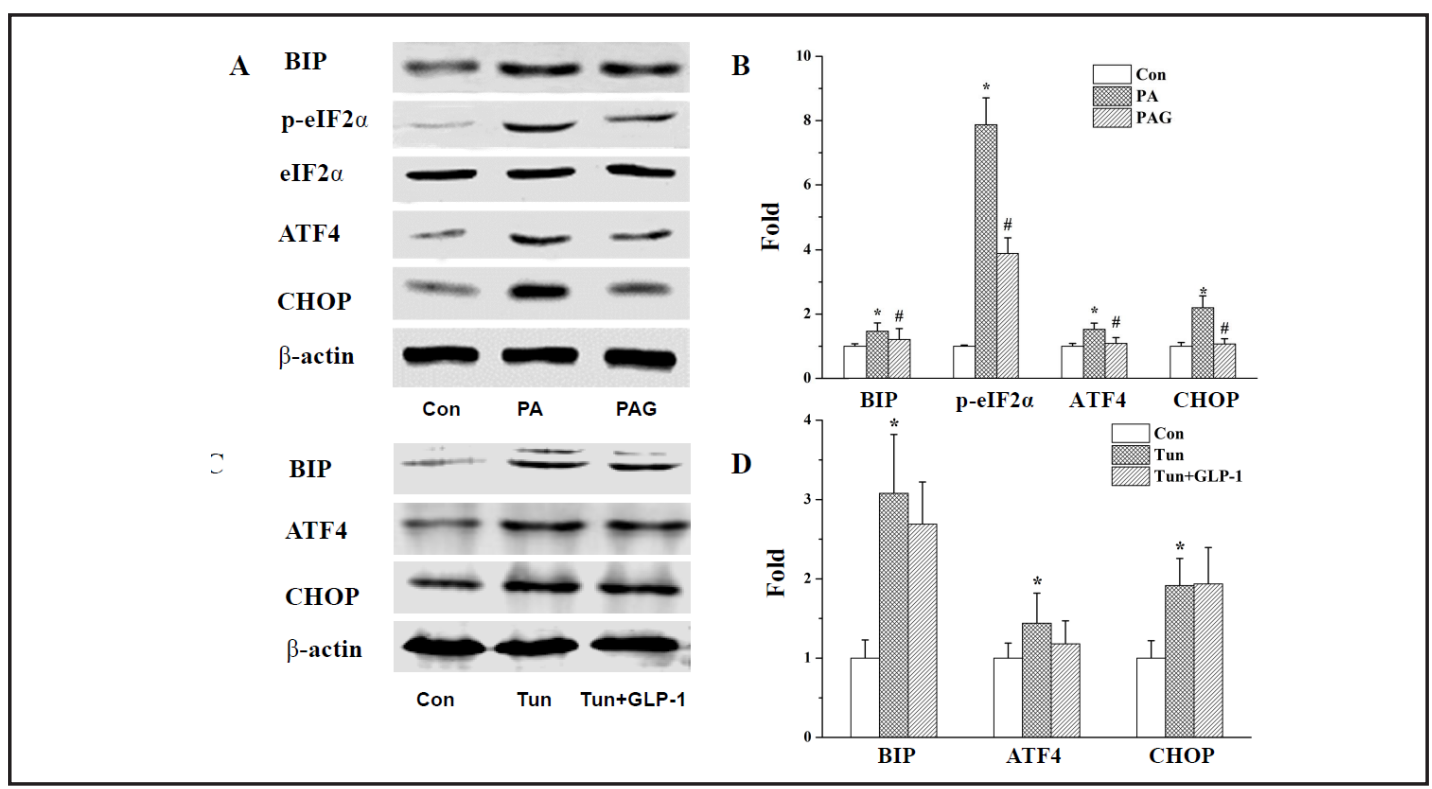

Fig. 6. GLP-1 treatment decreased ERS in HK2 cells induced by palmitic acid. A. Western blots analysis of ERS markers(BIP, p-eIF2 $\alpha$, ATF4 and CHOP proteins) of the palmitic acid infusion, were attenuated by GLP-1 treatment. B. Corresponding densitometric analyses of levels of ERS markers normalized to $\beta$-actin. C. Western blots analysis of tunicamycin induced increased ERS in the HK2 cells, which were not ameliorated by GLP-1 treatment. D. Corresponding densitometric analyses of levels of ERS protein normalized to $\beta$-actin. * $p<0.05$ compared with Con; ${ }^{\#} p<0.05$ compared with PA. Tun:Tunicamycin.

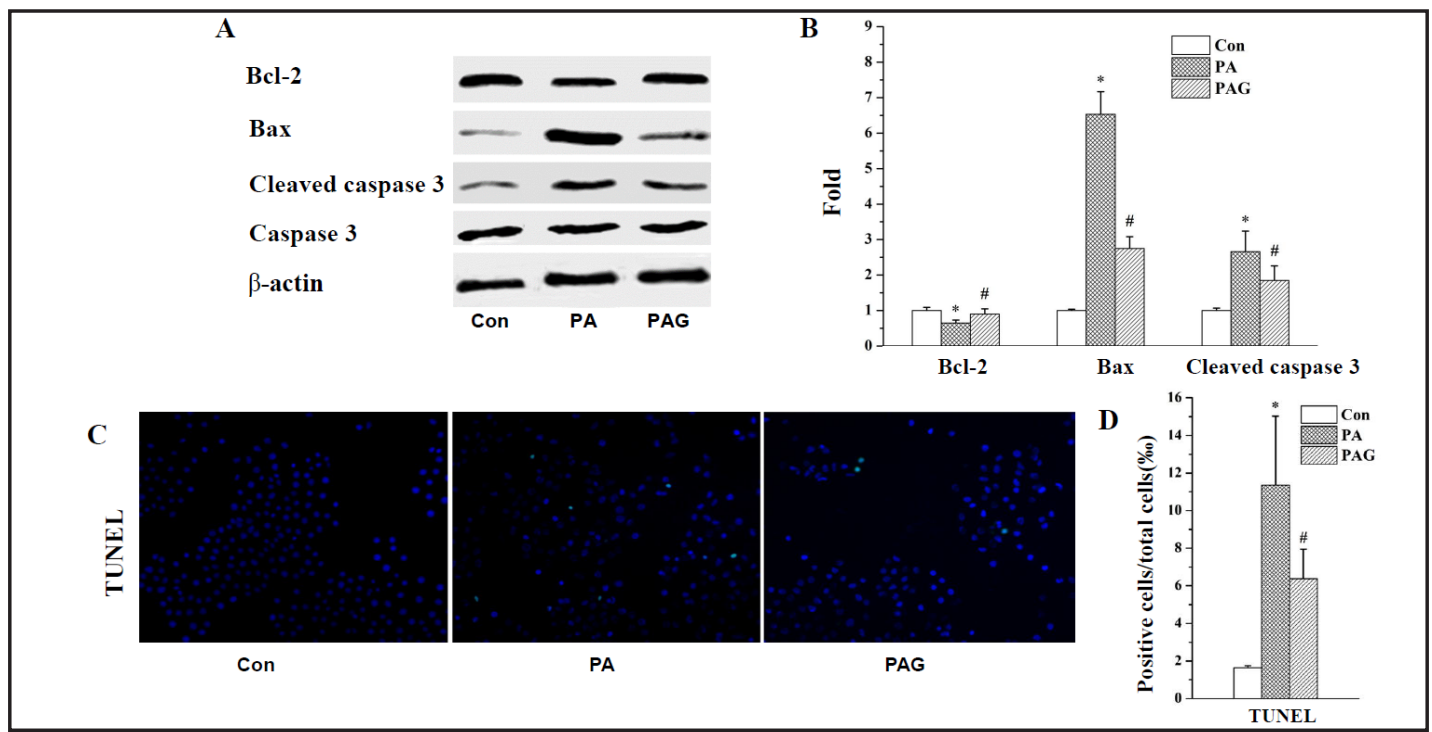

Fig. 7. GLP-1 treatment attenuated palmitic acid-induced HK2 cell apoptosis. A. Western blots analysis of the palmitic acid-induced decreased Bcl-2, and increased Bax and cleaved caspase-3 protein levels in HK2 cells, which were attenuated by GLP-1 treatment. B. Corresponding densitometric analyses of levels of Bcl2 , Bax and cleaved caspase-3 protein normalized to $\beta$-actin. $C$ and D. Representative TUNEL staining of HK2 cells. Apoptotic nuclei were observed. The percentage of apoptotic cells (TUNEL-positive cells) was obtained at least 10 fields from the three groups. Corresponding quantitative analysis indicated that GLP-1 treatment decreased the apoptotic HK2 cells. $p<0.05$ compared with Con; ${ }^{*} p<0.05$ compared with PA.

reduced by GLP-1 treatment in HFDG compared with HFD mice (Fig. 5C and D). In contrast, the level of angiotensin II was not significantly different between HFD and HFDG mice ( $p>0.05)$, as shown in Fig. 5E.

\section{KARGER}



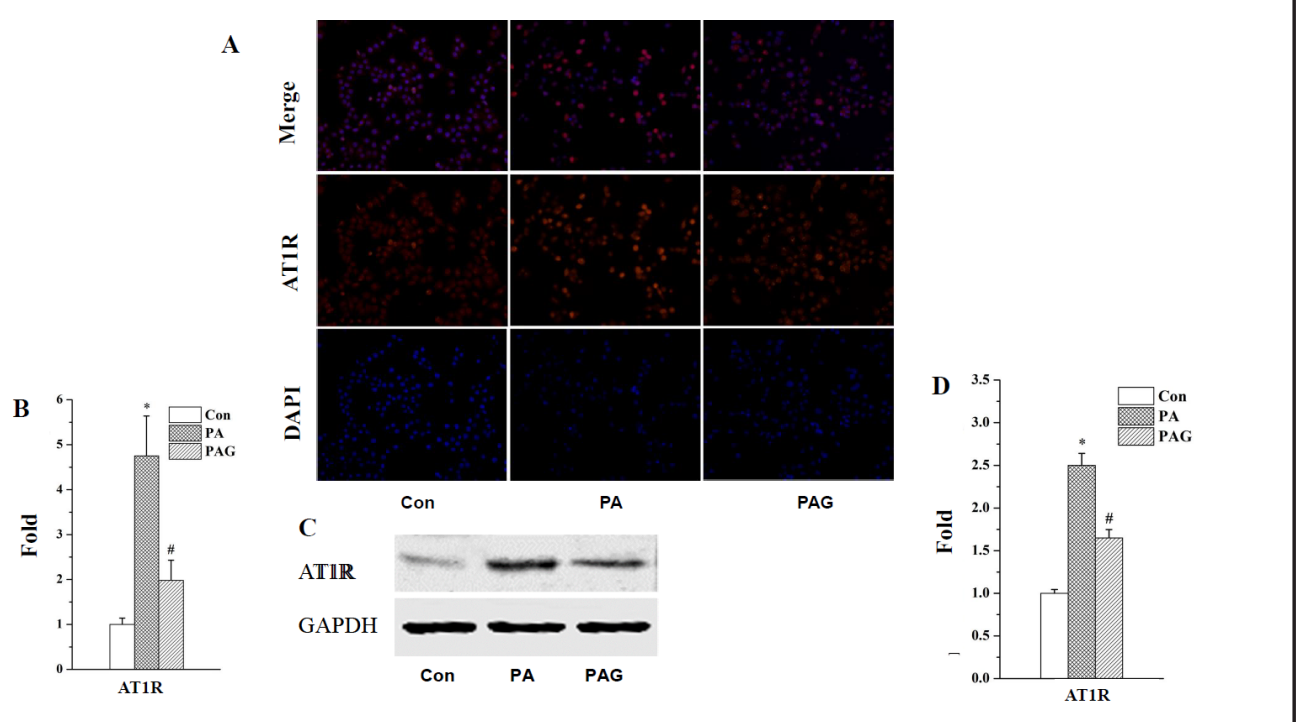

Fig. 8. GLP-1 treatment blocks AT1R expression in HK2 cells induced by palmitic acid. A. Immunofluorescence staining of AT1R in cultured HK2 cells. B. Quantitative analysis of AT1R positive staining. C. Western blots analysis of palmitic acid-induced increased AT1R expression in the HK2 cells, which were decreased by GLP-1 treatment. D. Corresponding densitometric analyses of levels of AT1R protein normalized to GAPDH. ${ }^{*} p<0.05$ compared with Con; ${ }^{\#} p<0.05$ compared with PA.

\section{GLP-1 prevented HK2 cell ERS and apoptosis induced by PA}

Incubation with PA increased ERS marker expression, including BIP, p-eIF2 $\alpha$, ATF4, and CHOP abundance, indicating that PA induced HK2 cell apoptosis. This was significantly prevented by GLP-1 (Fig. 6A and B). However, GLP-1 treatment could not prevent ERS from Tunicamycin in HK2 cells (Fig. 6C and D). Increased Bax and cleaved caspase 3 protein in HK2 induced by PA was reduced after GLP-1 treatment (Fig. 7A and B). The anti-apoptosis protein, Bcl-2, was improved by GLP-1 treatment after PA intervention. The percentage of HK2 cells containing TUNEL-positive nuclei markedly increased in HFD mice, which was attenuated significantly by GLP-1 treatment (Fig. 7C and D).

\section{GLP-1 blocked AT1R expression in HK2 cells induced by PA}

Following incubation of HK2 cells with PA, AT1R expression was dramatically increased, as indicated by western blotting and immunofluorescence (Fig. 8). HK2 cells incubated by PA was associated with significantly increased AT1R expression relative to Con cells $(p<0.05)$. The expression of AT1R was dramatically reduced with GLP-1 treatment compared to PAtreated cells.

However, GLP-1 treatment could not block AT1R expression and ameliorate apoptosis induced by up-regulation of AT1R with an over-expression plasmid AT1R-pEX-3 containing the full-length AT1R gene in PA-incubated HK2 cells (Fig. 9).

\section{Discussion}

The current study demonstrated the development and progression of HFD induced renal injury in mice, and GLP-1 treatment ameliorated the severity of injury related to increased SGLT2 expression, decreased ERS, and apoptosis. Also, GLP-1 treatment decreased the body weight with an accompanying reduction in food intake, and had a positive effect on SBP control. These findings were associated with a reduction in renal tubular AT1R expression.

The high-fat diet mouse model is characterized by hyperlipidemia and insulinemia as a result of obesity. Accumulating evidence demonstrated that obesity is associated with renal 


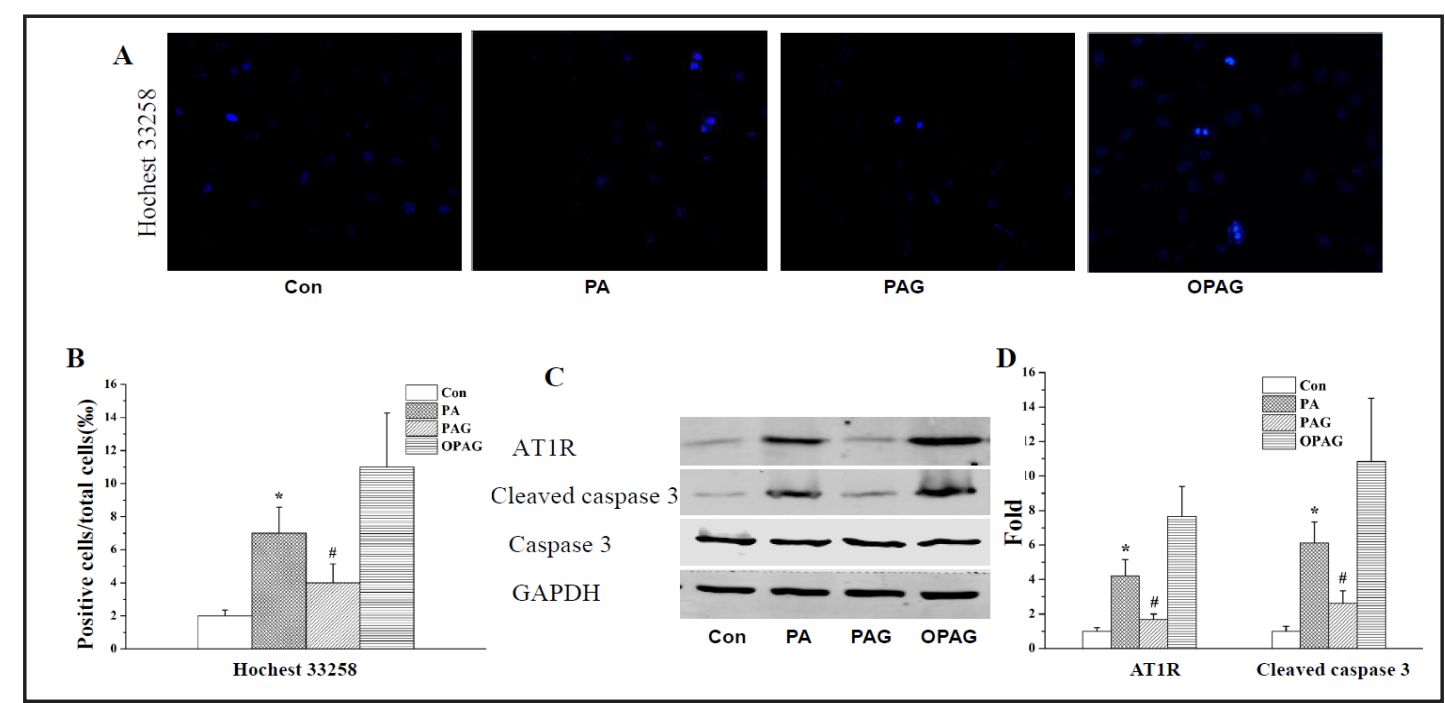

Fig. 9. GLP-1 treatment could not reduce apoptosis induced by over-expression of AT1R. A and B. Representative Hochest 33258 staining of HK2 cells. Apoptotic nuclei were observed. The percentage of apoptotic cells was obtained at least 10 fields from the three groups. Corresponding quantitative analysis indicated that GLP-1 treatment did not decrease the apoptotic HK2 cells in OPAG. C. Western blots analysis showed increased cleaved caspase-3 protein levels in HK2 cells with an over-expression plasmid AT1R-pEX-3 incubated by the palmitic acid, which were not attenuated by GLP-1 treatment. D. Corresponding densitometric analyses of levels of cleaved caspase- 3 protein normalized to caspase 3 . * $p<0.05$ compared with Con; \# $p<0.05$ compared with PA and OPAG. OPAG:over-expression of AT1R+PAG.

insufficiency in mice and humans [2,3]. We found vacuolated tubular cells completely filled in the cortex of the HFD mice in PAS and less extensive SGLT2 expressed in HFD mice compared with Con mice in the proximal tubule demonstrated by immunohistochemistry and western blotting. SGLT2 is located only in the apical brush border of the S1 and S2 portion of the proximal renal tubule. The SGLT2 reabsorbs approximately $90 \%$ of the filtered glucose load. Loss of expression of SGLT2 restricts renal function [20]. Decreased SGLT2 expression in HFD mice indicated that HFD induced proximal renal tubule injury, while GLP-1 treatment attenuated the disorder. Fatty acids have been proved to activate ERS in animal and human mesangial cells, podocytes, proximal tubular cells, and the tubulointerstitium [3, 21, 22]. ERS contributed to the pathophysiology of renal injury according to aberrant metabolic conditions, such as hyperglycemia and hyperlipidemia [3]. Our data confirmed that HFD mice and HK2 cells incubated with PA had induced significant ERS markers, such as BIP, p-eIF2 $\alpha$, ATF4, and CHOP. Additionally, the CHOP-mediated activation of apoptotic pathways was related to up-regulated Bax and down-regulated $\mathrm{Bcl}-2$, and increased expression of cleaved caspase 3 were involved in PA-induced tubule cells apoptosis both in vivo and in vitro.

GLP-1R is produced in the pancreas, gut, hypothalamus, and kidney. Previous studies demonstrated that GLP-1R played an anti-apoptosis role in INS-1 cells [23], H9c2 cells [24] and astrocytes [15]. GLP-1 binding to GLP-1R resulted in body weight loss due to decreased food intake. Weight loss improved metabolism relative to insulin sensitivity, hypertension, hyperlipidemia, and hyperglycemia [16]. The present study demonstrated the anti-hypertensive effects in HFDG mice followed by less AT1R expression. Blocking AT1R expression by valsartan decreases HFD-induced hypertension in mice [3]. Hence, we drew the conclusion that blocking AT1R expression by GLP-1 was responsible for the anti-hypertensive effects. The intake of abundant saturated fatty acids resulted in ERS in the intestines and decreased GLP-1 production [25], suggesting that exogenous GLP-1 may reduce the injury. GLP-1 receptor analog remarkably decreased markers of inflammation, oxidative stress, and fibrosis in the kidneys of maternal obesity mice [26]. GLP-1 decreased BIP and p-eIF2 $\alpha$ protection against ER stress-induced cell apoptosis-associated transcription 
factor CHOP in pancreatic INS-1 cells in Akita mice [23], while Cunha et al. [27] reported that GLP-1R treatment increased ATF4-CHOP expression in vitro. The discrepancy may be associated with temporal changes (i.e., acute $v s$. chronic effects). This study demonstrated that GLP-1 can alleviate ERS and apoptosis in the kidney of high-fat diet-induced mice, while GLP-1 treatment could not reduce ERS induced by tunicamycin in HK2 cells. Saturated fatty acids decreased Bcl-2, while GLP-1 treatment restored Bcl-2 protein compared to control levels in vivo and in vitro. Lipotoxic renal tubular cell apoptosis was prevented by enhancing cellular defense mechanisms via up-regulation of Bcl-2. GLP-1 treatment also decreased Bax and inhibited apoptosis, as evidenced by the lowered levels of cleaved caspase 3 and fewer TUNEL-positive cells compared to renal tubular cells exposed to saturated fatty acids. The current study showed that AT1R involved in renal injuries of mice on HFD, and blocking AT1R by GLP-1 treatment ameliorated injury in the renal tubules. Angiotensin II combined with AT1R contributed to PA-induced ERS and apoptosis in HK2 cells. The angiotensin II level in cultured medium increased after PA treatment. PA-induced up-regulation of ERS markers and apoptosis can be significantly reduced by AT1R blocker valsartan or renin inhibitor aliskiren, providing evidence for AT1R involved in ERS and apoptosis of kidney cells during increased fatty acid overload [3]. Angiotensin II binding increased level of AT1 receptor involved in activation of local renin-angiotensin system(RAS) in the kidney during increased fatty acid overload, which has been proved to induce kidney injury via ERS and apoptosis induced by saturated fatty acid infused in vitro and in vivo [3]. Serum angiotensin II levels significantly increased in HFD mice compared with controls while HFDG mice induced a higher level of angiotensin II than non-treated mice, but with no significant difference, which may be attributed to the blocked AT1R expression in HFDG mice. GLP-1 reversed glomerular endothelium cell injury through inhibition of angiotensin II signaling on cRaf (Ser259), supporting the view that GLP-1 primarily affected kidney physiology through inhibition of RAS activity in vitro [28]. In HFD and HK2 cells treated with PA, AT1R expression was inhibited after GLP-1 treatment. Previous studies reported that blocking AT1R prevents obesity-induced hypertension [3], insulin resistance [29], inflammation [29], fatty liver, and fibrosis [30] in obese animal models. Up-regulation of AT1R with an over-expression plasmid AT1R-pEX-3 in PA-incubated HK2 cells induced apoptosis was not ameliorated by GLP-1 treatment, while reduced AT1R expression in HFDG mice compared to HFD mice indicated GLP-1 treatment may exert renoprotective effects of saturated fatty acid-induced kidney tubular cell ERS and apoptosis, at least partly, by inactivation local RAS with blocking AT1R expression in vivo and in vitro. Healthy human treatment with a 2-h GLP-1 infusion appeared as a rapid decrease in plasma angiotensin II concentration, whereas renin, aldosterone, and the urinary excretion of angiotensinogen showed no significant changes [31]. This acute effect of GLP-1 on plasma angiotensin II concentration may not be in parallel to long-term GLP-1 infusion in mice, while the potential relationship between GLP-1 and AT1R warrant further study.

GLP-1 analogues are potentially useful for protecting against the detrimental effects in mice exposed to a high fat diet on renal. One clinical study with a focus on obesity indicated that a GLP-1 infusion reduced the glomerular filtration rate from 151 to $142 \mathrm{ml} / \mathrm{min}$, as measured by creatinine clearance [32], while more clinical studies are needed to validate the encouraging results.

\section{Acknowledgements}

This work was supported by grants from the National Natural Science Foundation of China, China 81270822 (to Yong Gu), 81570661 (to Yong Gu), 81300590 (to Wei Ding), 81400714(to Min Yang), and the Major State Basic Research Development Program of China 2012 CB517700 (to Yong Gu). 


\section{Cellular Physiology Cell Physiol Biochem 2017;41:1113-1124

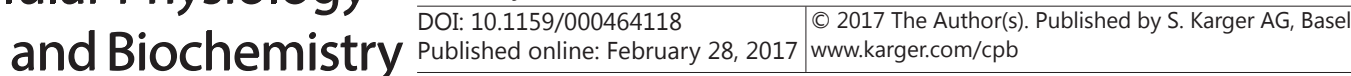 \\ Guo et al.: GLP-1 Ameliorates Renal Injury}

\section{Disclosure Statement}

There is no conflicts of interest.

\section{References}

1 de Vries AP, Ruggenenti P, Ruan XZ, Praga M, Cruzado JM, Bajema IM, D'Agati VD, Lamb HJ, Pongrac Barlovic D, Hojs R, Abbate M, Rodriquez R, Mogensen CE, Porrini E: Fatty kidney: Emerging role of ectopic lipid in obesity-related renal disease. Lancet Diabetes Endocrinol 2014;2:417-426.

-2 Park CW, Kim HW, Ko SH, Lim JH, Ryu GR, Chung HW, Han SW, Shin SJ, Bang BK, Breyer MD, Chang YS: Longterm treatment of glucagon-like peptide-1 analog exendin-4 ameliorates diabetic nephropathy through improving metabolic anomalies in db/db mice. J Am Soc Nephrol 2007;18:1227-1238.

- 3 Li C, Lin Y, Luo R, Chen S, Wang F, Zheng P, Levi M, Yang T, Wang W: Intrarenal renin-angiotensin system mediates fatty acid-induced er stress in the kidney. Am J Physiol Renal Physiol 2016;310:F351-363.

-4 Felizardo RJ, da Silva MB, Aguiar CF, Camara NO: Obesity in kidney disease: A heavyweight opponent. World J Nephrol 2014;3:50-63.

5 Gomez P, Ruilope LM, Barrios V, Navarro J, Prieto MA, Gonzalez O, Guerrero L, Zamorano MA, Filozof C: Prevalence of renal insufficiency in individuals with hypertension and obesity/overweight: The fath study. J Am Soc Nephrol 2006;17:S194-200.

6 Ejerblad E, Fored CM, Lindblad P, Fryzek J, McLaughlin JK, Nyren O: Obesity and risk for chronic renal failure. J Am Soc Nephrol 2006;17:1695-1702.

7 Cao J, Dai DL, Yao L, Yu HH, Ning B, Zhang Q Chen J, Cheng WH, Shen W, Yang ZX: Saturated fatty acid induction of endoplasmic reticulum stress and apoptosis in human liver cells via the PERK/ATF4/CHOP signaling pathway. Mol Cell Biochem 2012;364:115-129. Ma Y, Hendershot LM: The unfolding tale of the unfolded protein response. Cell 2001;107:827-830. Sieber J, Lindenmeyer MT, Kampe K, Campbell KN, Cohen CD, Hopfer H, Mundel P, Jehle AW: Regulation of podocyte survival and endoplasmic reticulum stress by fatty acids. Am J Physiol Renal Physiol 2010;299:F821-829.

10 Cooper R, Forrester T, Ogunbiyi O, Muffinda J: Angiotensinogen levels and obesity in four black populations. Icshib investigators. J Hypertens 1998;16:571-575.

11 Chu KY, Leung PS: Angiotensin II in type 2 diabetes mellitus. Curr Protein Pept Sci 2009;10:75-84.

12 Tsubouchi H, Inoguchi T, Inuo M, Kakimoto M, Sonta T, Sonoda N, Sasaki S, Kobayashi K, Sumimoto H, Nawata H: Sulfonylurea as well as elevated glucose levels stimulate reactive oxygen species production in the pancreatic beta-cell line, min6-a role of NAD(P)H oxidase in beta-cells. Biochem Biophys Res Commun 2005;326:60-65.

-13 Kouyama R, Suganami T, Nishida J, Tanaka M, Toyoda T, Kiso M, Chiwata T, Miyamoto Y, Yoshimasa Y, Fukamizu A, Horiuchi M, Hirata Y, Ogawa Y: Attenuation of diet-induced weight gain and adiposity through increased energy expenditure in mice lacking angiotensin II type 1a receptor. Endocrinology 2005;146:3481-3489.

-14 Lee YS, Park MS, Choung JS, Kim SS, Oh HH, Choi CS, Ha SY, Kang Y, Kim Y, Jun HS: Glucagon-like peptide-1 inhibits adipose tissue macrophage infiltration and inflammation in an obese mouse model of diabetes. Diabetologia 2012;55:2456-2468.

15 Bao Y, Jiang L, Chen H, Zou J, Liu Z, Shi Y: The neuroprotective effect of liraglutide is mediated by glucagon-like peptide 1 receptor-mediated activation of cAMP/PKA/CREB pathway. Cell Physiol Biochem 2015;36:2366-2378.

16 Zander M, Madsbad S, Madsen JL, Holst JJ: Effect of 6-week course of glucagon-like peptide 1 on glycaemic control, insulin sensitivity, and beta-cell function in type 2 diabetes: A parallel-group study. Lancet 2002;359:824-830.

17 Docherty NG, le Roux CW: Improvements in the metabolic milieu following Roux-en-y gastric bypass and the arrest of diabetic kidney disease. Exp Physiol 2014;99:1146-1153.

18 Katagiri D, Hamasaki Y, Doi K, Okamoto K, Negishi K, Nangaku M, Noiri E: Protection of glucagonlike peptide-1 in cisplatin-induced renal injury elucidates gut-kidney connection. J Am Soc Nephrol 2013;24:2034-2043. 


\section{Cellular Physiology Cell Physiol Biochem 2017;41:1113-1124

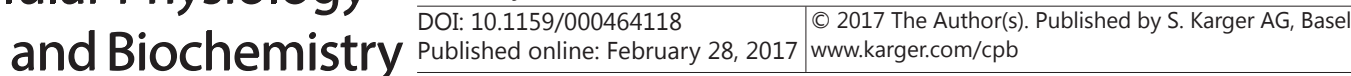 \\ Guo et al.: GLP-1 Ameliorates Renal Injury}

19 Nistala R, Habibi J, Aroor A, Sowers JR, Hayden MR, Meuth A, Knight W, Hancock T, Klein T, DeMarco VG, Whaley-Connell A: Dpp4 inhibition attenuates filtration barrier injury and oxidant stress in the Zucker obese rat. Obesity (Silver Spring) 2014;22:2172-2179.

-20 Ly JP, Onay T, Sison K, Sivaskandarajah G, Sabbisetti V, Li L, Bonventre JV, Flenniken A, Paragas N, Barasch JM, Adamson SL, Osborne L, Rossant J, Schnermann J, Quaggin SE: The sweet pee model for SGLT2 mutation. J Am Soc Nephrol 2011;22:113-123.

21 Lim JC, Lim SK, Han HJ, Park SH: Cannabinoid receptor 1 mediates palmitic acid-induced apoptosis via endoplasmic reticulum stress in human renal proximal tubular cells. J Cell Physiol 2010;225:654-663.

-22 Katsoulieris E, Mabley JG, Samai M, Green IC, Chatterjee PK: Alpha-linolenic acid protects renal cells against palmitic acid lipotoxicity via inhibition of endoplasmic reticulum stress. Eur J Pharmacol 2009;623:107112.

23 Zhao L, Guo H, Chen H, Petersen RB, Zheng L, Peng A, Huang K: Effect of liraglutide on endoplasmic reticulum stress in diabetes. Biochem Biophys Res Commun 2013;441:133-138.

24 Jiang YQ, Chang GL, Wang Y, Zhang DY, Cao L, Liu J: Geniposide prevents hypoxia/reoxygenation-induced apoptosis in H9C2 cells: Improvement of mitochondrial dysfunction and activation of GLP-1R and the PI3K/AKT signaling pathway. Cell Physiol Biochem 2016;39:407-421.

25 Hayashi H, Yamada R, Das SS, Sato T, Takahashi A, Hiratsuka M, Hirasawa N: Glucagon-like peptide-1 production in the glutag cell line is impaired by free fatty acids via endoplasmic reticulum stress. Metabolism 2014;63:800-811.

26 Glastras SJ, Chen H, McGrath RT, Zaky AA, Gill AJ, Pollock CA, Saad S: Effect of GLP-1 receptor activation on offspring kidney health in a rat model of maternal obesity. Sci Rep 2016;6:23525.

-27 27 Cunha DA, Ladriere L, Ortis F, Igoillo-Esteve M, Gurzov EN, Lupi R, Marchetti P, Eizirik DL, Cnop M: Glucagon-like peptide-1 agonists protect pancreatic beta-cells from lipotoxic endoplasmic reticulum stress through upregulation of BIP and JunB. Diabetes 2009;58:2851-2862.

-28 Premaratna SD, Manickam E, Begg DP, Rayment DJ, Hafandi A, Jois M, Cameron-Smith D, Weisinger RS: Angiotensin-converting enzyme inhibition reverses diet-induced obesity, insulin resistance and inflammation in C57BL/6J mice. Int J Obes (Lond) 2012;36:233-243.

29 Toblli JE, Munoz MC, Cao G, Mella J, Pereyra L, Mastai R: ACE inhibition and AT1 receptor blockade prevent fatty liver and fibrosis in obese Zucker rats. Obesity (Silver Spring) 2008;16:770-776.

-30 Mima A, Hiraoka-Yamomoto J, Li Q, Kitada M, Li C, Geraldes P, Matsumoto M, Mizutani K, Park K, Cahill C, Nishikawa S, Rask-Madsen C, King GL: Protective effects of GLP-1 on glomerular endothelium and its inhibition by PKC $\beta$ activation in diabetes. Diabetes 2012;61:2967-2979.

-31 Skov J, Dejgaard A, Frokiaer J, Holst JJ, Jonassen T, Rittig S, Christiansen JS: Glucagon-like peptide-1 (GLP1): Effect on kidney hemodynamics and renin-angiotensin-aldosterone system in healthy men. J Clin Endocrinol Metab 2013;98:E664-671.

-32 Gutzwiller JP, Tschopp S, Bock A, Zehnder CE, Huber AR, Kreyenbuehl M, Gutmann H, Drewe J, Henzen C, Goeke B, Beglinger C: Glucagon-like peptide 1 induces natriuresis in healthy subjects and in insulinresistant obese men. J Clin Endocrinol Metab 2004;89:3055-3061. 